\title{
Too Sanitary for Vultures
}

José A. Donázar; Antoni Margalida; Martina Carrete; José A. Sánchez-Zapata

The current crisis of biodiversity has hit old-world vultures especially hard; populations that flourished in the mid-20th century over much of Asia and Africa are in some cases close to extinction. In Europe, however, vultures have been spared, and today the Mediterranean basin is home to the largest populations of the Western Palearctic. The availability of carcasses around stock farms became a decisive factor in the survival and expansion of vulture populations in southern Europe.

The outbreak of bovine spongiform encephalopathy (BSE) in 2001 led to the passing of sanitary legislation (Regulation CE 1774/2002) that greatly restricted the use of animal byproducts not intended for human consumption. All carcasses of domestic animals had to be collected from farms and transformed or destroyed in authorized plants, contradicting member states' obligations and efforts to conserve scavenger species. The effects of this policy include a halt in population growth, a decrease in breeding success, and an apparent increase in mortality of young age classes. Conservation concerns determined the appearance of a number of European dispositions (2003/322/CE 2005/830/CE) regulating the use of animal byproducts as food for necrophagous birds. Although the aim of these dispositions is to guarantee food supplies, in practice the spatial distribution of feeding stations has become almost totally predictable and habitat quality has been modified artificially as a result. The repercussions of these changes on individuals, populations, and communities of avian scavengers are undoubtedly significant.

Partial solutions to this problem are being found, albeit slowly. In 2009, the European Union began discussing a Spanish proposal for a revised regulation of the use of animal byproducts not intended for human consumption. In April 2009, amendments were approved by the European Parliament obliging the European Commission to regulate exceptions that will guarantee the supply of carcasses and thus satisfy the requirements of avian scavenger populations. It is hoped that the definitive legislation will be in place by 2010 to 2011. The philosophy of this new policy should allow member states a greater flexibility in carrion provisioning. Taking into account sanitary guarantees, it seems clear that encouraging fallen stock to be left in situ is the most ecologically harmonious, inexpensive, and efficient management method for the conservation of scavengers. Fortunately, current knowledge of scavenger ecology is increasing rapidly and so, more than ever, collaboration between ecologists, veterinarians, farmers, and legislators is both possible and desirable 